\title{
Viscoelasticity of amyloid plaques in transgenic mouse brain studied by Brillouin microspectroscopy and correlative Raman analysis
}

\author{
Sara Mattana*, Silvia Caponi ${ }^{\dagger}$, Francesco Tamagnini*, \\ Daniele Fioretto* and Francesca Palombo ${ }^{\S, \boldsymbol{\uparrow}}$ \\ *Department of Physics and Geology \\ University of Perugia, Perugia I-06123, Italy \\ ${ }^{\dagger}$ Istituto Officina dei Materiali del CNR (CNR-IOM) - Unità di Perugia \\ Department of Physics and Geology \\ University of Perugia, Perugia I-06123, Italy \\ \$ Hatherly Laboratories, Medical School \\ University of Exeter, Exeter EX4 $4 P S, U K$ \\ ${ }^{\S}$ School of Physics and Astronomy \\ University of Exeter, Exeter EX4 4QL, UK \\ qf.palombo@exeter.ac.uk
}

Received 1 February 2017

Accepted 16 March 2017

Published 21 April 2017

\begin{abstract}
Amyloidopathy is one of the most prominent hallmarks of Alzheimer's disease (AD), the leading cause of dementia worldwide, and is characterized by the accumulation of amyloid plaques in the brain parenchyma. The plaques consist of abnormal deposits mainly composed of an aggregationprone protein fragment, $\beta$-amyloid 1-40/1-42, into the extracellular matrix. Brillouin microspectroscopy is an all-optical contactless technique that is based on the interaction between visible light and longitudinal acoustic waves or phonons, giving access to the viscoelasticity of a sample on a subcellular scale. Here, we describe the first application of micromechanical mapping based on Brillouin scattering spectroscopy to probe the stiffness of individual amyloid plaques in the hippocampal part of the brain of a $\beta$-amyloid overexpressing transgenic mouse. Correlative analysis based on Brillouin and Raman microspectroscopy showed that amyloid plaques have a complex structure with a rigid core of $\beta$-pleated sheet conformation ( $\beta$-amyloid) protein surrounded by a softer ring-shaped region richer in lipids and other protein conformations. These preliminary results give a new insight into the plaque biophysics and biomechanics, and a valuable contrast mechanism for the study and diagnosis of amyloidopathy.
\end{abstract}

Keywords: Alzheimer's; imaging; vibrational spectroscopy; protein misfolding; light scattering.

TCorresponding author.

This is an Open Access article published by World Scientific Publishing Company. It is distributed under the terms of the Creative Commons Attribution 4.0 (CC-BY) License. Further distribution of this work is permitted, provided the original work is properly cited. 


\section{S. Mattana et al.}

\section{Introduction}

Alzheimer's disease (AD) is the most common form of dementia, affecting approximately 47 million people worldwide with an incidence that is predicted to double every twenty years owing to ageing population. ${ }^{1}$ Amyloidopathy, which is characterized by abnormal deposits of an aggregation-prone polypeptide, amyloid beta $(\mathrm{A} \beta)$, within the parenchyma of AD brain, is one of the two most common hallmarks of $\mathrm{AD}$, the other one being tauopathy. The A $\beta$ polypeptide is composed of $40-42$ amino acids with prevalent $\beta$-pleated sheet conformation; through hydrophobic interactions and hydrogen bonding, it tends to form a range of aggregates, from dimers to oligomers of various sizes, which can phase-separate from the aqueous medium of the intact brain and give rise to extracellular deposits or 'plaques'.

Amyloidopathy has been regarded, in the last 30 years, as the main underlying cause in the pathogenesis of $\mathrm{AD},{ }^{2}$ and has been associated to alterations in cognitive and neurophysiological function, ${ }^{3}$ both at a neural network ${ }^{4}$ and single cell level. ${ }^{5-8}$

Previous work using a combination of microFourier Transform Infrared (FTIR) spectroscopic imaging, Raman microscopy and immunostaining has shown the complex structure and biochemistry of $\mathrm{A} \beta$ plaques in the hippocampal Cornu Ammonis 1 (CA1) region of the brain in a genetically engineered mouse, the TASTPM model (F. Palombo et al., manuscript in preparation). This mouse presents, at the age of 9-12 months, severe accumulation of amyloid plaques. The plaque has a dense core of predominantly $\beta$-pleated sheet conformation protein surrounded by a ring-shaped region richer in lipid esters and other protein conformations. While its origin is still debated, this lipid structure constitutes an amphiphilic 'interface' between the essentially hydrophobic core and the hydrophilic medium surrounding the plaque.

In this work, we applied Brillouin microspectroscopy and correlative Raman scattering to investigate amyloid plaques in cryosections of TASTPM hippocampus, a brain area critically involved in memory encoding. Brillouin spectroscopy is an alloptical contactless technique for the viscoelastic characterization of biological materials. Micromechanical information is obtained by shining visible laser light onto a sample and measuring the inelastic light scattering from acoustic waves or phonons causing a shift in frequency of the light in the $\mathrm{GHz}$ region (hypersounds). Spatial resolution, which is diffraction limited to approximately $300 \mathrm{~nm}$ according to Abbe criterion using visible light, allows one to single out viscoelastic heterogeneities on a subcellular scale. We previously applied this technique to study the biomechanics of protein fibers (collagen and elastin) of the extracellular matrix obtaining the full determination of their elastic constants,,${ }^{9,10}$ and to map the elasticity of epithelial tissue biopsy in ex vivo sections of Barrett's oesophagus. ${ }^{11,12}$ The same technique, through the use of single etalon (VIPA) approaches, has been applied to other clinically relevant samples such as keratoconus ${ }^{13}$ and ageing crystalline lens, ${ }^{14}$ bacterial meningitis in cerebrospinal fluid, ${ }^{15}$ and atherosclerotic mouse carotid artery. ${ }^{16}$

Traditionally, Brillouin spectroscopy has been performed using a scanning Fabry-Perot (FP) type interferometer, which gives very high contrast and spectral resolution at the expenses of the scanning time (sometimes minutes for a single spectrum). An angle-dispersive FP interferometer has also been used to demonstrate for the first time a Brillouin imaging modality. ${ }^{17}$ Alternative approaches to the use of the multipass FP interferometer for Brillouin scattering analysis have recently been developed based on multistage virtually image phased array (VIPA) spectrometers ${ }^{18}$ which enable rapid mapping at the expenses of achievable contrast which is key to analyze turbid media such as biomedical samples. Further advances in this direction are required to make Brillouin microspectroscopy a viable technology for rapid diagnostics in the context of healthcare and clinical applications. In those cases whereby high contrast and high resolution are required for a full viscoelastic characterization of a sample, multipass FP interferometers are still the preferred choice. Indeed, the Brillouin spectrum of biological matter derived from an FP-type spectrometer presents Brillouin bandshapes that are less affected by the interferometer's response function and can be reproduced by full viscoelastic functions ${ }^{19,20}$ or, at least, by damped harmonic oscillator functions around the Brillouin peaks. ${ }^{21,22}$ These high-contrast high-resolution spectra are better suited for accurate viscoelastic analysis of materials than those derived from VIPA-type spectrometers, hence making it possible to determine the acoustic wave attenuation and apparent viscosity (from the Brillouin linewidth). 
Here, we present the detailed viscoelastic characterization of amyloid plaques in transgenic mouse brain by Brillouin microspectroscopy with a tandem multipass FP interferometer, and correlative microRaman analysis to provide the molecular structure and composition in correspondence to the mechanics of the plaques. Viscoelastic properties of Alzheimer's brain are expected to be different from those of healthy brain, owing to biochemical and biophysical changes underlying neurodegenerative disease. Preliminary results of the correlative analysis based on site-matched Brillouin and Raman microspectroscopy presented in this work unveil changes in mechanical properties due to amyloidopathy which can become a contrast mechanism for diagnosis of neuropathology. This is the first application of Brillouin scattering to dementia-related problems and will potentially pave the way to future developments in healthcare technology applied to the field of neurophysiology.

\section{Materials and Methods}

\subsection{Sample preparation}

This work was carried out in accordance with the UK Home Office Guidelines and the University of Exeter Animal Welfare Ethical Review Board. 12 -month old A $\beta$-overexpressing TASTPM transgenic male mice were used in this study. TASTPM carries two mutations on the gene for the amyloid precursor protein (APPSwe K670N, M671L) and one on the presenilin 1 gene (M146V) that can be found in patients affected by familiar AD. ${ }^{23,24}$ All animals were housed at room temperature under a $12 / 12$ hour light/dark cycle, with free access to food and water ad libitum before being sacrificed. ${ }^{7}$ The brain was rapidly removed and horizontal acute slices of $300 \mu \mathrm{m}$ thickness were cut in a vibratome and suspended in artificial cerebrospinal fluid, before use for electrophysiological recordings. A number of slices of each brain containing hippocampus, striatum and cortex were retained for the present study. The slices were post-fixed overnight with $4 \%$ formalin $+0.1 \mathrm{M}$ phosphate buffer solution (PBS). This enabled to store the slices indefinitely while preventing deterioration from exposure to room temperature conditions. Note that, although this can affect the mechanical properties of tissues, the use of a fixative is critical for structural and conformational analysis performed by Raman scattering here. Nevertheless, this effect acts uniformly on the tissue, changing in the same way the elastic properties of the healthy and the plaque zones. Previous work has shown that the cross-links that form in the process of formalin fixation 'lock in' the secondary structure of protein molecules; ${ }^{25}$ therefore, proteins retain the secondary structure present before fixation, i.e., the structure that confers them a particular rigidity (see below). The slices were rinsed twice with $0.1 \mathrm{M}$ PBS (5 min each time) and stored in $30 \%(\mathrm{w} / \mathrm{v})$ sucrose solution to inhibit subsequent formation of ice crystals, before being embedded in a water-soluble frozen section medium (NEG-50, Thermo Scientific) and snap frozen. Sections of $20 \mu \mathrm{m}$ thickness were cut in a cryostat and left to rinse for at least $24 \mathrm{~h}$ in $0.1 \mathrm{M}$ PBS. Sections were subsequently rinsed in distilled water and mounted onto calcium fluoride slides (Crystran). Two plaques within a brain hippocampal section of TASTPM mouse were analyzed, and a total of three microspectroscopic maps were obtained.

\subsection{Brillouin microspectroscopy}

Micro-Brillouin maps were collected with an FPbased mapping system depicted in Fig. 1. A laser beam from a $532 \mathrm{~nm}$ single-mode solid state laser is focused onto the sample by a customized JRS Scientific Instruments CM-1 Confocal Microscope. A polarizing beam splitter is placed in the beam path to transmit the depolarized backscattered light towards the spectrometer. Pinholes at the entrance of the (3+3)-pass FP interferometer provide a confocal arrangement for microscopic mapping. A Mitutoyo long working distance $20 \times$ (NA 0.42 ) objective is used for both focusing the laser beam on and collecting the backscattered light from the sample. A $\lambda / 4$ wave plate is inserted upstream of the objective to switch from depolarized to unpolarized scattering configuration. The sample is mounted onto an xyz microtranslation stage for mapping acquisition.

The backscattered light is dispersed through a tandem (3+3)-pass FP interferometer onto a single photon avalanche photodiode. Spectra were acquired in the range -30 to $30 \mathrm{GHz}$ using a $90 \mathrm{~s}$ exposure time to achieve a reasonable signal-to-noise ratio, with a $5 \mathrm{~mW}$ laser power on the sample. GHOST software was used for data acquisition and processing. ${ }^{26}$ Accurate focus adjustment of the objective was performed before each series of measurements. This gave approximately $2 \mu \mathrm{m}$ for the 


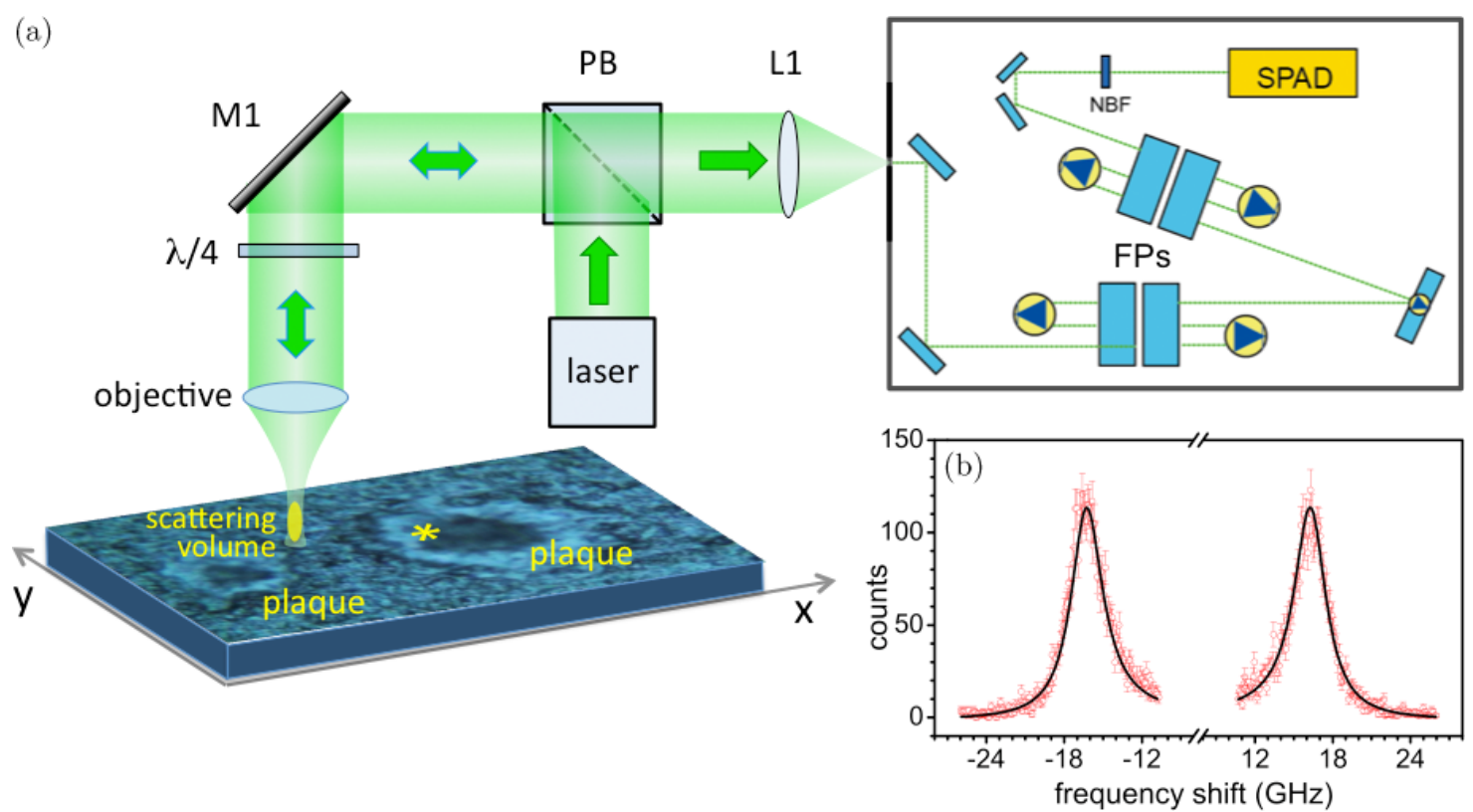

Fig. 1. (a) Schematic of the micro-Brillouin experiment conducted on a TASTPM mouse brain hippocampal section containing two A $\beta$ plaques. (Photomicrograph was obtained using a Renishaw inVia Raman microscope with $20 \times$ objective.) M1: mirror; PB: polarizing beam splitter; L1: lens; FPs: Fabry-Perot interferometers; NBF: narrow bandpass filter; SPAD: single photon avalanche photodiode. (b) Micro-Brillouin spectrum extracted from the scattering volume indicated by an asterisk in (a). Results from fit analysis to a damped harmonic oscillator function are shown (peak position: $16 \mathrm{GHz}$ ).

lateral and $8 \mu \mathrm{m}$ for the axial dimension of the scattering volume. The role of different spatial scales involved in micro-Brillouin measurements is described in Sec. 2.2.1.

A typical spectrum obtained from a single microBrillouin measurement of TG mouse hippocampus (CA1 region) is reported in Fig. 1(b). This shows both (Stokes and anti-Stokes) symmetric branches of the Doppler-shifted Brillouin spectrum. The Brillouin peaks at approximately $16 \mathrm{GHz}$ are due to longitudinal acoustic modes propagating within the sample at the position of the scattering volume shown in the photomicrograph (Fig. 1(a)). Curve-fit analysis based on a damped harmonic oscillator (DHO, Eq. (2) below) model was applied to the Brillouin peak to extract the values of frequency position $\left(\omega_{b}\right)$ and linewidth $\left(\Gamma_{b}\right)$, which are related to the viscoelastic properties of the sampled volume, i.e., the real and imaginary parts of the longitudinal elastic modulus, respectively. ${ }^{27}$ Viscoelasticity of soft matter in the $\mathrm{GHz}$ spectral range is briefly recalled in Sec. 2.2.2.

\subsubsection{Relevant spatial length scales for Bio- Brillouin scattering measurements}

Brillouin scattering from inhomogeneous media such as biomedical samples is a quite complex matter because the presence of discontinuities can profoundly affect propagation and attenuation of both light and acoustic waves. Here, we outline some basic features to better understand the results obtained in this work and, more generally, to start a discussion that deserves the attention of the Bio-Brillouin community.

Three different (subcellular) spatial scales, covering about three orders of magnitude in length, regulate the results of Brillouin scattering experiments performed on inhomogeneous samples: (i) the smallest one $\left(L_{1} \sim 0.1 \mu \mathrm{m}\right)$ is related to the wavelength of acoustic modes, (ii) the intermediate $\left(L_{2} \sim 1 \mu \mathrm{m}\right)$ to the attenuation of acoustic modes, and (iii) the largest one $\left(L_{3} \sim 10 \mu \mathrm{m}\right.$ in micro-Brillouin measurements) to the size of the scattering volume (Fig. 2).

\section{$\mathrm{L}_{1}$ : Wavelength of acoustic modes}

In backscattering geometry (Fig. 2(a)), the wavelength of acoustic modes $\Lambda$ is related to the wavelength of the excitation laser $\lambda$ through the relation: $\Lambda=\lambda / 2 n \sim 0.18 \mu \mathrm{m}$, where $n$ is the refractive index of the sample material. Inhomogeneity on a length scale which is much smaller than this is hidden to the acoustic field and hence an effective 


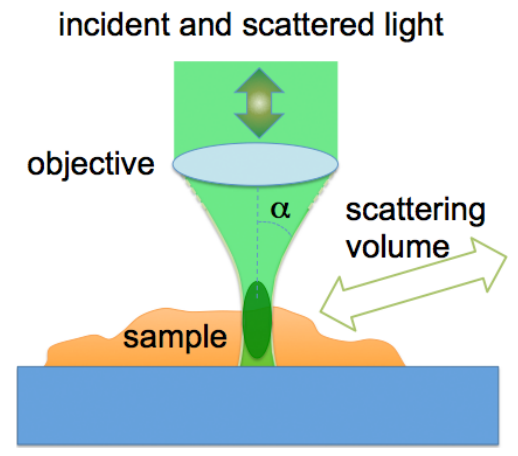

(a)

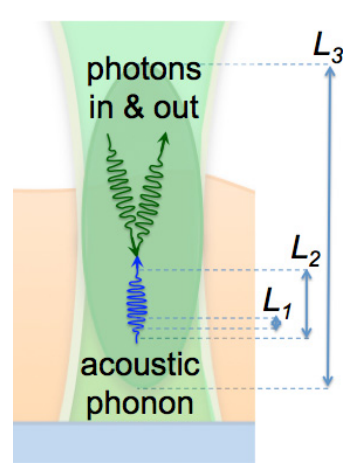

(b)
Fig. 2. (a) Schematic diagram of the micro-Brillouin scattering experiment. (b) Close-up view of the interaction between an incoming light beam and an acoustic phonon within the scattering volume, giving rise to the Brillouin scattering effect. $L_{1}$, $L_{2}$ and $L_{3}$ denote the relevant length scales in this interaction.

homogeneous medium is revealed, with average elastic constants. ${ }^{28,29}$

However, the case of inhomogeneity on a length scale approaching $L_{1} / 10$ is the most challenging one to deal with, since acoustic scattering effects can become dominant, giving rise to anomalous dispersion and attenuation of acoustic modes (see, for instance, Refs. [30-31]). Analysis of these phenomena is beyond the aim of the present work.

For inhomogeneous media on length scales larger than $L_{1}$, an important role is played by $L_{2}$ and $L_{3}$, which can induce an inhomogeneous broadening of Brillouin peaks that should be distinct from homogeneous effects in order to gain the viscoelastic characterization of the sample.

\section{$\mathrm{L}_{2}$ : Attenuation of acoustic modes}

The Brillouin doublet is due to light scattered by longitudinal acoustic phonons, i.e., density fluctuations $\rho_{q}(t)$ propagating with wavevector $q$ which, in backscattering geometry, is given by $q=2 n k_{i}$, where $k_{i}$ is the wavevector of the incident light and $n$ the refractive index of the sample. Some contribution can be expected also from lower $q$ values, due to the finite aperture of the objective, as previously reported for Brillouin imaging ${ }^{32}$ and explained below for the present experiments. In the case of simple homogeneous liquids, the linearized hydrodynamic equations, neglecting the thermal diffusion mode and its contribution to the acoustic damping, give:

$$
\left[\left(\frac{\rho}{q^{2}}\right) \frac{\partial^{2}}{\partial t^{2}}+\eta_{0} \frac{\partial}{\partial t}+M_{0}\right] \rho_{q}(t)=0,
$$

where $\rho$ is the static mass density, $M_{0}=\rho c_{0}^{2}$ is the adiabatic longitudinal modulus, $c_{0}$ the adiabatic sound velocity, and $\eta_{0}$ the static longitudinal viscosity, responsible for the damping of acoustic waves.

Looking for harmonic solutions, $\rho_{q}(t) \propto \exp (i \omega t)$, the presence of a complex elastic modulus $M(\omega)=$ $M_{0}+i \omega \eta_{0}$ becomes apparent in Eq. (1). Though more sophisticated models for $M(\omega)$ should be applied in the case of viscoelastic media, as shown in Sec. 2.2.2, this simple approximation for $M(\omega)$ is able to describe propagation and attenuation of density fluctuations in liquids (simple hydrodynamics), solids (Voigt model of viscoelasticity ${ }^{33,34}$ and even in general viscoelastic media, provided that a narrow frequency region around the Brillouin peaks is analyzed ${ }^{35}$ and apparent, $\omega$-dependent values of $M\left(\omega_{b}\right)$ and $\eta\left(\omega_{b}\right)$ are defined. In this condition, the spectrum of scattered light is that of a damped harmonic oscillator (DHO):

$$
I(\omega)=\frac{I_{0}}{\pi} \frac{\Gamma_{b} \omega_{b}^{2}}{\left(\omega^{2}-\omega_{b}^{2}\right)^{2}+\left(\Gamma_{b} \omega\right)^{2}},
$$

where $\omega_{b}$ and $\Gamma_{b}$ approximately corresponds to the frequency position and full width at half maximum of the Brillouin peak. These parameters are related to the (apparent) longitudinal modulus and (apparent) viscosity through: $M\left(\omega_{b}\right)=\rho \omega_{b}^{2} / q^{2}$, and $\eta\left(\omega_{b}\right)=\rho \Gamma_{b} / q^{2}$.

Internal friction can also be obtained from these parameters through the inverse of the quality factor of the Brillouin peak, $Q^{-1}=\Gamma_{b} / \omega_{b}$. The quality factor can be intuitively defined as the number of oscillations of the system before their amplitude decreases by a factor $e$, so that the characteristic propagation length of acoustic phonons, their mean free path, can be estimated as $L_{2} \sim 2 \pi Q / q^{36}$ For the spectrum in Fig. 1(b), we obtained $\omega_{b} / \Gamma_{b} \sim 5$, corresponding to $L_{2} \sim 1 \mu \mathrm{m}$.

Alternatively, a Lorentzian function can be used to fit Brillouin peaks. In that case, a further function must be added to the Lorentzian to account for the asymmetry and the frequency shift of the peaks. ${ }^{37}$ Instead Eq. (2) includes all these effects and needs no additional manipulation.

It is worth noting that fitting the experimental spectra to Eq. (2) gives the correct values of $\omega_{b}$ and $\Gamma_{b}$, provided that spurious broadening effects are adequately addressed. Firstly, Eq. (2) requires convolution with the instrumental function, i.e., the 


\section{S. Mattana et al.}

measured spectrum of monochromatic light, to yield the experimental spectral band-shape. Moreover, the asymmetric broadening generated by the finite angle of collection of scattered light ( $\alpha$ in Fig. 2(a)) needs to be taken into account. Though this effect may be quite complex, ${ }^{38}$ in the backscattering geometry used in Brillouin microscopy the broadening is minimized and the collected $q$ values are mainly ranging between $q_{M}=2 n k_{i}$ and $q_{m}=2 n k$ sin $[(\pi-\alpha) / 2]$, neglecting the contribution from incident light far from the optical axis. The induced broadening of Brillouin peaks can approximately be estimated by the relationship $\Delta \omega_{b} / \omega_{b}=\Delta q / q$, where $\Delta q=q_{M}-q_{m}$. In our case, having used an objective with NA 0.42 gives a $\sim 2 \%$ broadening, a rather small effect which can be included into the convolution process as a small enlargement of the instrumental function and $\mathrm{a} \sim 1 \%$ reduction of the average $q$ value. In the case of larger numerical apertures or scattering geometries different from the backscattering, this contribution may become dominant and the fit to a single DHO function must be replaced by a distribution of functions, with the appropriate weight in $q .{ }^{38}$

Other spurious effects which can distort the Brillouin lines are the absorption in strongly opaque media, which can induce an indetermination in the value of $q$, and multiple scattering from turbid media, which is a source of inelastic scattering at smaller $q$ values and gives a broadening of the measured Brillouin peaks towards lower frequencies. A detailed analysis of these effects is beyond our aims. The effects of multiple scattering in Brillouin spectra from biological matter is discussed in a further work (M. Mattarelli et al., manuscript in preparation).

\section{$\mathrm{L}_{3}$ : Scattering volume}

The scattering volume $L_{3}$ may be larger than $L_{2}$, being a potential origin for inhomogeneous broadening of Brillouin lines.

In micro-Brillouin measurements, the size of the scattering volume is determined both by the shape of the incident light beam and by the diameter of the pinhole that defines the confocal condition. The scattering volume can operatively be defined as the region of the enlightened sample from which scattered light enters the pinhole and, passing through the interferometer, then reaches the photo-detector.
It can be approximated to an ellipsoid, several times as long as it is wide. In our set-up, the width is approximately $2 \mu \mathrm{m}$ and the length is $8 \mu \mathrm{m}$ (S. Caponi et al., manuscript in preparation). This is the smallest portion of the sample that can be investigated, defining the granularity of the final Brillouin map. By increasing the NA and reducing the diameter of the pinhole, $L_{3}$ can be reduced by almost one order of magnitude ${ }^{39}$ at the expenses of reducing the scattered light intensity by nearly $2-3$ orders of magnitude, at a fixed power density of the incident light. Note that the value of the power density cannot be arbitrarily increased, being limited to the amount that starts causing photo-damage to the sample.

Within a single scattering volume, a distribution of homogeneous sub-regions larger than $L_{2}$ can be present, each one characterized by a given elastic constant. In this case, the measured spectrum is the sum of Brillouin peaks originating from the different sub-regions. If their spectral spacing is larger than their width (after convolution with the instrumental function), their contribution can be singled out and their relative scattering intensity can give information on the relative volume of the sub-regions. Otherwise, a heterogeneous broadening is measured and $\omega_{b}$ is an average of those of the subunits. This may be the origin of the uncorrelated (broad) peak width and frequency shift observed (see below) in the region of the extracellular matrix surrounding the lipid ring.

\subsubsection{Viscoelastic behavior of biological samples}

When mapping viscoelastic materials such as cells and tissues, different values of probed Brillouin frequencies cannot immediately relate to stronger or weaker interactions between molecules, i.e., to genuine changes in elastic properties of the sample, as a potential increase or decrease in viscosity should also be taken into account. In fact, small changes of, e.g., humidity in different parts of the sample can give appreciable differences in acoustic wave velocity, mainly due to a change of viscosity and of the corresponding structural relaxation time, rather than to a change in bonds strength. Here, some basic properties of viscoelasticity are recalled, which can help the interpretation of viscoelastic maps of biological samples. 
In molecular liquids and soft matter, density fluctuations may be coupled with molecular internal degrees of freedom and the structural relaxation (or $\alpha$-relaxation) process, giving rise to dispersion and absorption of acoustic modes. ${ }^{19,40}$ These relaxation effects which are responsible for the order of magnitude difference between elastic moduli probed at Brillouin frequencies and in quasi-static conditions can be taken into account by a generalization of the hydrodynamic equations, introducing a complex $\omega$-dependent elastic modulus $M(\omega)=$ $M^{\prime}(\omega)+i M^{\prime \prime}(\omega)$ which can be expressed as

$$
M(\omega)=M_{\infty}+\Delta M(\omega)+i \omega \eta_{\infty},
$$

where $M_{\infty}=\rho c_{\infty}^{2}$ is the high-frequency (solid-like) longitudinal modulus and $\eta_{\infty}$ is the high frequency longitudinal viscosity. $\Delta M(\omega)$ can be arbitrarily complex, depending on the number and nature of the relaxation processes that are coupled with density fluctuations. The simplest possible scenario is depicted in Fig. 3: it shows a single relaxation, the $\alpha$-relaxation, which is present in all viscoelastic materials and is usually described by a ColeDavidson relaxation function ${ }^{41}$

$$
\Delta M(\omega)=\left(M_{0}-M_{\infty}\right) /(1+i \omega \tau)^{\beta},
$$

where $\tau$ is the characteristic relaxation time and $\beta$ is the stretching parameter, describing the deviation

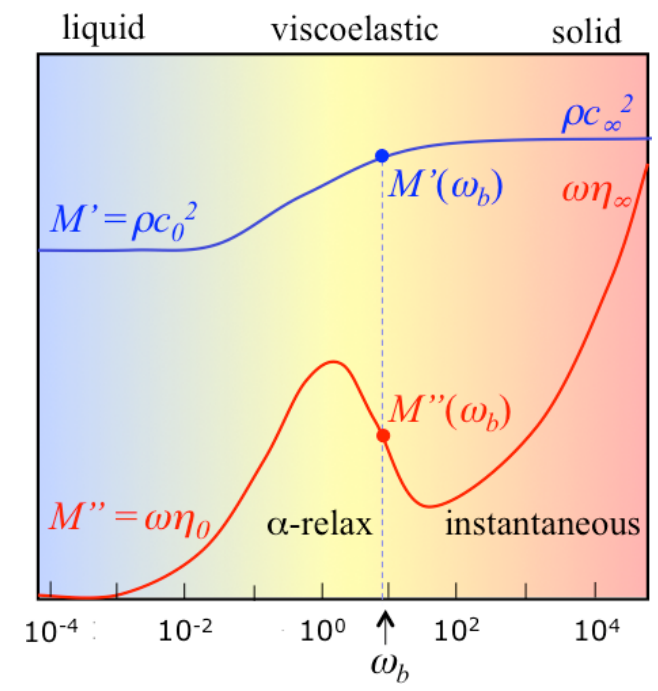

Fig. 3. Sketch of the simplest possible scenario for the frequency dependence of the real $\left(M^{\prime}\right)$ and imaginary $\left(M^{\prime \prime}\right)$ parts of the elastic modulus in a viscoelastic medium, above the glass transition temperature. Frequency is normalized to that of the $\alpha$-relaxation, the boundary between liquid-like and solid-like behavior. from a single exponential behavior (see Ref. 27 and references therein).

Note that in the $\omega \tau \ll 1$ limit (corresponding to, e.g., low frequency phonons or high temperature or high hydration of the sample), it results that $\Delta M(\omega)=\left(M_{0}-M_{\infty}\right)+i \omega \tau\left(\eta_{0}-\eta_{\infty}\right)$, where $\eta_{0}-\eta_{\infty}=$ $\left(M_{\infty}-M_{0}\right) \tau$, recalling the Maxwell model for stress relaxation. This limit matches with the simple hydrodynamics $M(\omega)=M_{0}+i \omega \eta_{0}$ expressed by Eq. (1), corresponding to the low frequency part of Fig. 3. If the Brillouin peak lies in this region, an increase of the acoustic mode velocity can occur with an increase of static viscosity $\eta_{0}$ or relaxation time $\tau$ ( $\alpha$-relaxation moving towards lower frequencies in Fig. 3) due to, e.g., a reduction of humidity in the sample. In this case, an increase of frequency shift correlates with an increase of linewidth of Brillouin peaks. We have found such a behavior in highly hydrated tissues, namely cornea samples (unpublished results).

The opposite case, $\omega \tau \gg 1$, corresponds to $\Delta M=0$ and $M(\omega)=M_{\infty}+i \omega \eta_{\infty}$, the Voigt model of solids, represented in the high frequency part of Fig. 3.

The intermediate condition, the central region in Fig. 3, is typical of Brillouin scattering from highly viscous materials. If $\omega_{b}$ lies in this intermediate region, as depicted in the figure, an increase of frequency shift would correlate with a decrease of linewidth of the Brillouin peak, and vice versa.

This is the case of the lipid ring in the TASTPM sample (see Fig. 4). As a consequence, the lipid ring appears as a quite homogeneous part of the sample, at least on the $L_{2}$ scale, with a reduced static viscosity, whose origin is still debated and will be the focus of further investigation.

\subsection{Raman microspectroscopy}

Micro-Raman maps were collected with a Renishaw inVia Raman microscope using a near-infrared $830 \mathrm{~nm}$ laser and a Leica long working distance $50 \times($ NA 0.50$)$ objective. The backscattered light from the sample was analyzed by a spectrometer comprising a $600 \mathrm{gr} / \mathrm{mm}$ grating and a Renishaw CCD camera. Raman maps were acquired in streamline mode with an exposure time of $50 \mathrm{~s}$ per point in the 2356$384 \mathrm{~cm}^{-1}$ spectral region. WiRE 4.0 software was used for acquisition and manipulation of the data.

Spectral maps were corrected via cosmic ray removal with a nearest neighbor routine and then 
(a)

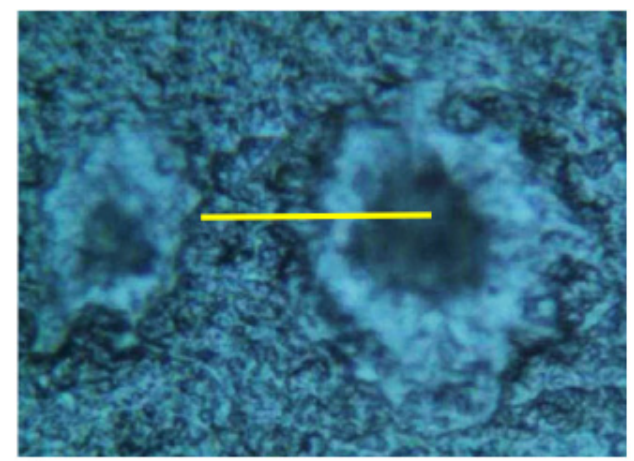

(b)

(c)

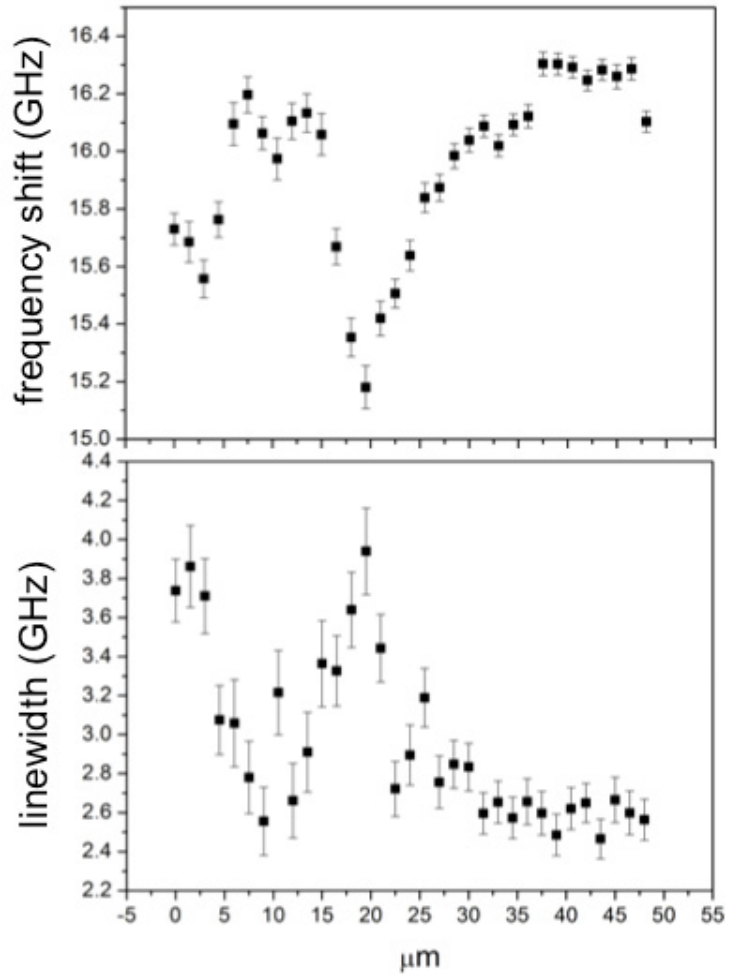

Fig. 4. (Color online) Photomicrograph and results of fit analysis applied to Brillouin spectra of a TASTPM mouse brain hippocampal section. (a) Photomicrograph obtained using a Renishaw inVia Raman microscope with $20 \times$ objective. (Yellow line denotes a $50 \mu \mathrm{m}$ long region, where Brillouin spectra were acquired through line scanning using a $1.5 \mu \mathrm{m}$ step-size.) Plots of the (b) frequency shift and (c) linewidth of the Brillouin peak derived from fit analysis of the spectra in the linear scan; error bars indicate the standard error (square root of number of counts).

analyzed by Principal Component Analysis (PCA) using 10 components, spectrum centring + normalization (SNV) pre-processing. In this manner, the first principal component (PC1) corresponds to the average spectrum of the sample in the mapped region. Results were reported as obtained, without any further modification including baseline correction.

\section{Results and Discussion}

\subsection{Micro-Brillouin mapping}

Figure 4(a) shows a photomicrograph of a TASTPM mouse brain hippocampal section containing two plaques. The plaques are visible as a dark core (rich in $\mathrm{A} \beta$ polypeptide) separated from the normal tissue by a lighter lipid ring. The molecular composition of the plaques was investigated through correlative chemical mapping based on Raman microscopy (see below).

A first series of Brillouin spectra was collected along a $50 \mu \mathrm{m}$ line scan starting from the periphery through to the center of a plaque (yellow line in Fig. 4(a)).

Plots of the frequency and linewidth of the Brillouin peak derived from fit analysis (to Eq. (2) in Sec. 2.2.1) of the spectra extracted from the line scan are reported in Figs. 4(b) and 4(c). The heterogeneous nature of the sample is evidenced by the position-dependent mechanical properties, highlighting the appropriateness of the microspectroscopic approach to investigate the mechanical differences between plaque, lipid ring and normal tissue. The Brillouin frequency shift (Fig. 4(b)) shows a decrease when going from the normal tissue to the lipid ring and a marked increase going towards the core of the plaque. To better understand the nature of this variation, it is useful to compare this behavior with the linear map of Brillouin linewidth reported in Fig. 4(c). Indeed it can be seen that $\Gamma_{b}$ has a maximum (broader peak) in the same region where $\omega_{b}$ has a minimum (smaller shift), corresponding to the lipid ring in Fig. 4(a). Such correlation between $\Gamma_{b}$ and $\omega_{b}$ is the characteristic signature of a viscoelastic effect, as detailed in Sec. 2.2.2 and previously observed in epithelial tissue, Barrett's oesophagus. ${ }^{11}$ Conversely, $\omega_{b}$ appears to be quite uncorrelated from $\Gamma_{b}$ in correspondence of the normal tissue, suggesting a heterogeneous origin for the broadening of Brillouin peaks therein.

A two-dimensional Brillouin map gives further evidence to these observations.

Figure 5(a) shows a microphotograph of the sample with a frame delimiting the region investigated by micro-Brillouin. The maps of Brillouin frequency shift and linewidth reported in Figs. 5(b) and $5(\mathrm{c})$ were obtained with a $1.5 \mu \mathrm{m}$ step raster scan of the sample, and spectra were fitted to the DHO model (Eq. (2) in Sec. 2.2.1). 
(a)

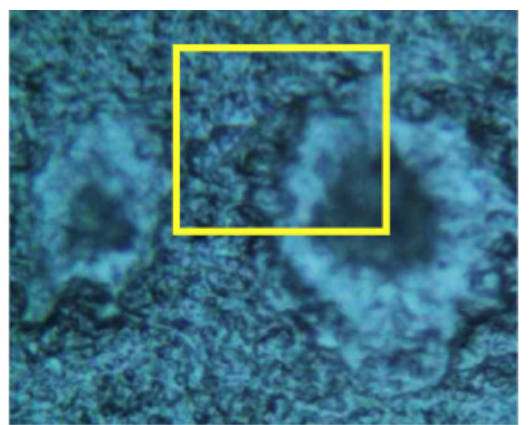

(b)

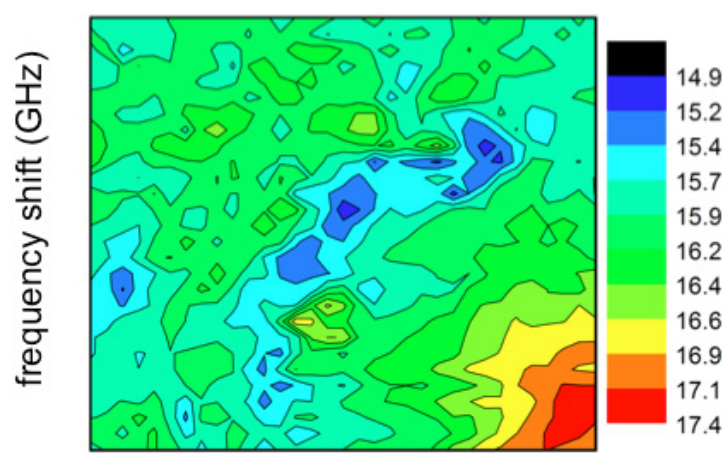

(c)

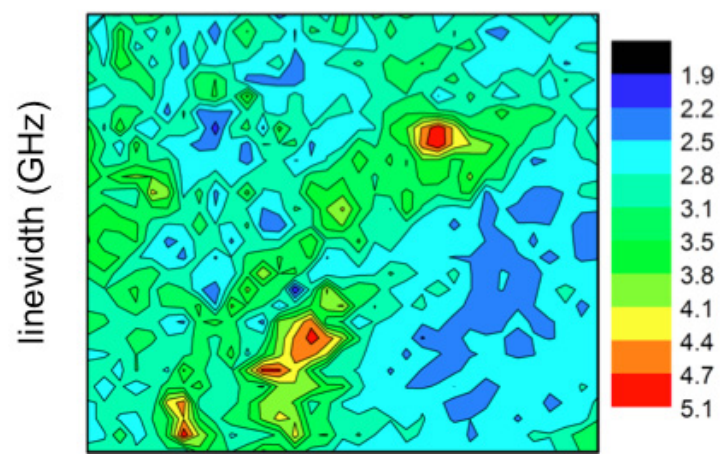

Fig. 5. (a) Photomicrograph of a TASTPM mouse brain hippocampal section containing two plaques. (Yellow box denotes a $50 \times 43 \mu \mathrm{m}^{2}$ area where a Brillouin map was acquired using a $1.5 \mu \mathrm{m}$ step-size.) Maps based on the (b) frequency shift and (c) linewidth of the Brillouin peak derived from fit analysis of the spectra in the map. Red color region in (b) denotes the plaque core, whilst in (c) it denotes the lipid-loaded ring surrounding the core.

Figure 5(b) shows a clear maximum in $\omega_{b}$ (higher rigidity) at the core of the plaque, approximately $10 \%$ larger than average, corresponding to $\sim 20 \%$ increase in elastic modulus (assuming constant density and refractive index). Distinct mechanical properties in correspondence to different types of protein secondary structure and conformational disorder have previously been identified. ${ }^{42}$ Secondary structures of proteins follow the increasing rigidity order: random coil $<\alpha$-helix $<\beta$-sheet. ${ }^{43}$ Hence, the rigidity of the plaque core observed here can be attributed to the deposition of aggregates of $\beta$-amyloid protein in $\beta$-pleated sheet conformation and to the exclusion of hydration water from this highly hydrophobic region.

Moving radially away from the core of the plaque towards the periphery, the Brillouin map of frequency shift shows (Fig. 5(b)) a decrease of $\omega_{b}$, with a minimum in the region of the lipid ring. It would be unwary to attribute this effect entirely to a reduction of stiffness, since a viscoelastic effect can also contribute to a decrease in apparent elastic modulus. In fact, in this area, the reduction of $\omega_{b}$ correlates quite strictly with an increase of $\Gamma_{b}$ (Fig. 5(c)), which can be explained in terms of a reduction of static viscosity and a blue-shift of the $\alpha$-relaxation process (see Fig. 3). Note that this counterintuitive effect, i.e., broadening of the Brillouin peak in correspondence to a decreasing static viscosity, requires a full-viscoelastic approach and cannot be accounted for neither in the liquid-like (simple hydrodynamic) or in the solid-like (Voigt) limit. A possible origin for the reduction of viscosity in the lipid ring can be the retention of hydration water within this amphiphilic region. Further investigation will be required to causally relate this mechanical behavior with the molecular composition of the ring.

The top left side of the maps in Figs. 5(b) and 5(c) corresponds to the normal tissue's extracellular matrix. In this region, $\Gamma_{b}$ markedly changes from one point to another showing no apparent correlation with $\omega_{b}$. In this case, heterogeneous broadening effects may be the cause for this behavior, as explained in Sec. 2.2.1. Heterogeneities in tissue can indeed be expected due to the presence of cell bodies (astrocytes and microglia) in the area surrounding the plaque - stratum radiatum of the CA1 subfield of the hippocampus.

The Brillouin peak frequency for the normal extracellular matrix (ECM) observed here (Figs. 4(b) and $5(\mathrm{~b}))$, approximately $16 \mathrm{GHz}$, is lower than the average frequency of the fibrous matrix of human epithelium biopsy (ca. $18 \mathrm{GHz}),{ }^{11}$ which in turn is lower than the frequency of type-I collagen fibers (ca. $19 \mathrm{GHz}) \cdot{ }^{9,10}$ This indicates that other noncollagenous constituents and the structural organization of the ECM contribute to giving rise to differences in $\omega_{b}$. Protein fibers of the ECM studied by quasi-static stress-strain testing show a Young's modulus in the MPa range (R. Edginton et al. manuscript in preparation). The presence of viscoelastic effects (Sec. 2.2.2) can induce 


\section{S. Mattana et al.}

order-of-magnitude increase of the elastic moduli measured in the $\mathrm{GHz}$ range with respect to those measured by quasi-static techniques. Though some works (see for instance Ref. 44) have reported the existence of a correlation between high and low frequency values of the moduli, this is a topic that deserves an in-depth phenomenological and theoretical investigation.

\subsection{Micro-Raman mapping}

Figure 6 shows the results of correlative Raman mapping on the same $\mathrm{A} \beta$ plaque analyzed by microBrillouin scattering.

Principal Component Analysis applied to the micro-Raman map provided the distribution of the plaque core (PC2; Fig. 6(b)) distinct from the lipid loaded ring (PC4; Fig. 6(c)). (Note that PC1 yielded the average spectrum of the specimen; data not shown.) Corresponding loading plots for these PCs are shown in Fig. 7.

The PC2 loading plot refers to the spectrum of the plaque core (Fig. 6(b)), with distinctive protein bands. The single Raman peak at $1663 \mathrm{~cm}^{-1}$ can be attributed to the amide I mode of protein with a $\beta$-pleated sheet conformation, which indeed is concentrated in the plaque core (Fig. 6(b)). Other notable bands are at $1441\left(\mathrm{CH}_{2}\right.$ bending) and around $1000 \mathrm{~cm}^{-1}$ (Phenylalanine). Instead, the PC4 spectrum presents a derivative-type signal in the amide $I$ region (with minimum at $1668 \mathrm{~cm}^{-1}$ and maximum at $1650 \mathrm{~cm}^{-1}$ ) which indicates that $\mathrm{PC} 4$ is a linear combination of spectra where positive signals corresponds to essentially lipids and other protein conformations, e.g., $\alpha$-helix which resonates at lower frequency than $\beta$-sheet in the amide I region. Other bands at 1435,1294 and $1059 \mathrm{~cm}^{-1}$ can be assigned to the $\mathrm{CH}_{2}$ deformation in protein and lipid, $\mathrm{CH}_{2}$ deformation in lipids, and skeletal $\mathrm{C}-\mathrm{C}$ stretching in lipids, respectively, in analogy with the assignment in Ref. 45. The absence of the Phenylalanine peak in PC4 indicates a different protein primary structure than PC1. Indeed, the PC4 loading plot relates to the lipid-rich layer surrounding the plaque core (Fig. 6(c)).

Therefore, correlative Brillouin-Raman mapping enables us to conclude that, in TASTPM mouse brain hippocampus, the $\mathrm{A} \beta$ plaque core has high rigidity identified by a marked increase in Brillouin peak frequency associated with the abnormal (a)

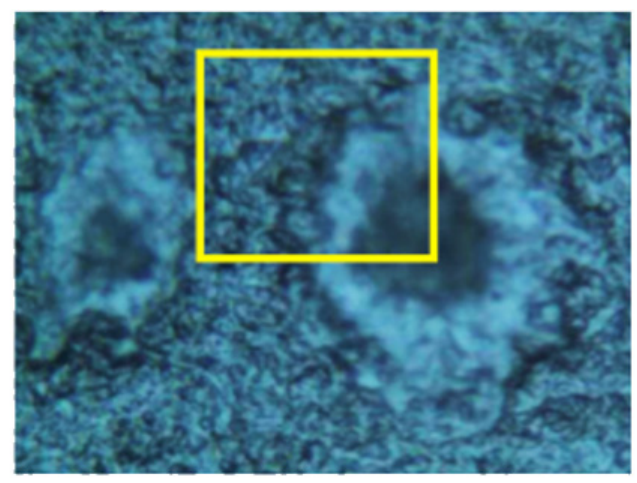

(b)

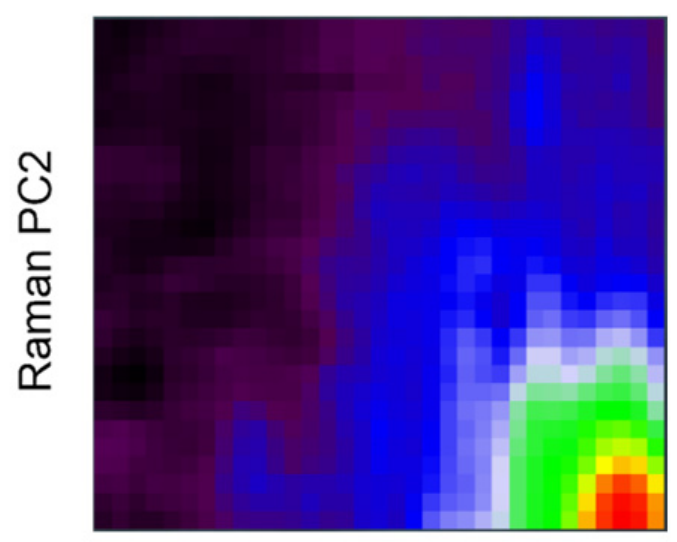

(c)

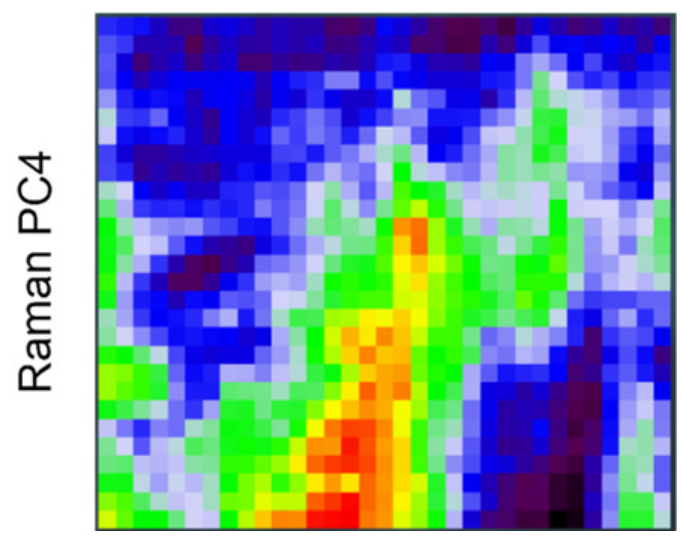

Fig. 6. (Color online) (a) Photomicrograph of a TASTPM mouse brain hippocampal section containing two plaques. (Yellow box denotes a $46 \times 39 \mu \mathrm{m}^{2}$ area where a Raman map was acquired using a $1.4 \mu \mathrm{m}$ step-size). (b, c) Map scores of the principal components (PCs) obtained from the analysis of the micro-Raman map. Red color region in (b) denotes the plaque core, whilst in (c) it denotes the lipid-loaded ring surrounding the core.

deposition of $\beta$-amyloid protein, which is a typical pathological hallmark of Alzheimer's disease. The surrounding tissue presents high viscoelasticity in correspondence of a lipid-rich layer around the dense core of the plaque and high heterogeneity in the external tissue, plausibly due to the presence of cell bodies (i.e., astrocytes and microglia). 


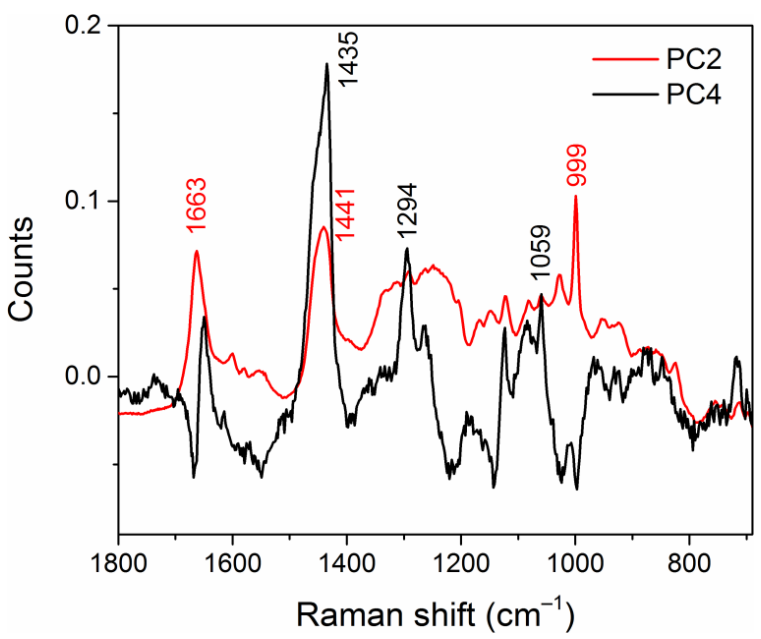

Fig. 7. Loading plots of the principal components (PCs) obtained from the analysis of a micro-Raman map of a TASTPM mouse brain hippocampal section. Peak positions of the main bands are labeled.

\subsection{Whole plaque viscoelasticity}

At a visual inspection, different plaques can show quite different morphologies due to their structural complexity. Therefore, we can expect that the viscoelastic behavior is also reflecting this heterogeneity.

Figure 8(a) shows the microphotograph of a plaque from a different area of the same mouse brain specimen. The maps of Brillouin frequency shift and linewidth are reported in Figs. 8(b) and 8(c). Also, here the core of the plaque is characterized by a distinctive maximum in $\omega_{b}$ (higher rigidity), approximately $10 \%$ larger than average, whilst the region around the plaque core shows a decrease of $\omega_{b}$, with a minimum (cyan-blue region in Fig. 8(b)) which correlates with a maximum in $\Gamma_{b}$ (red-yellow region in Fig. 8(c)), although this is less apparent than in Fig. 5(c).

For this plaque, the region of the lipid ring is visible but less defined than in the previous plaque (Fig. 5), hence indicating a differential distribution in plaque composition, structure and biomechanics across the TASTPM hippocampus.

Although an accurate evaluation of elastic modulus maps requires a point-by-point determination of density $\rho$ and refractive index $n$ of the sample, it can be noticed that $M^{\prime}=\rho \omega_{b}^{2} / q^{2}=\rho(\lambda \nu / 2 n)^{2}$ where $\nu$ is the Brillouin frequency shift, and $\lambda$ is the wavelength of laser light. Here the ratio $\rho / n^{2}$ is constant, with good approximation, far from
Viscoelasticity of amyloid plaques in transgenic mouse brain

(a)

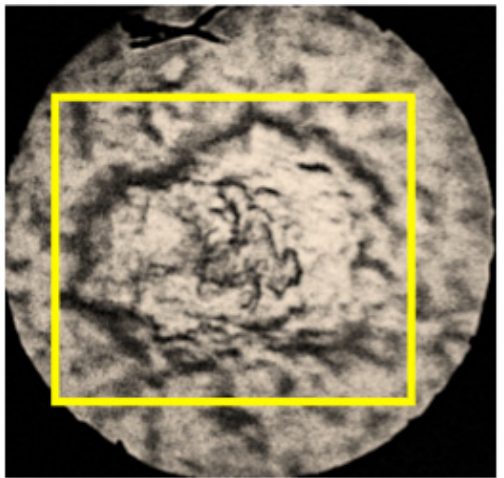

(b)

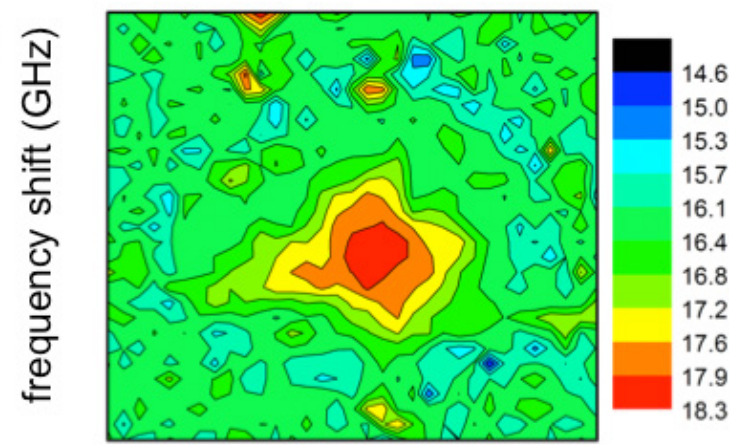

(c)

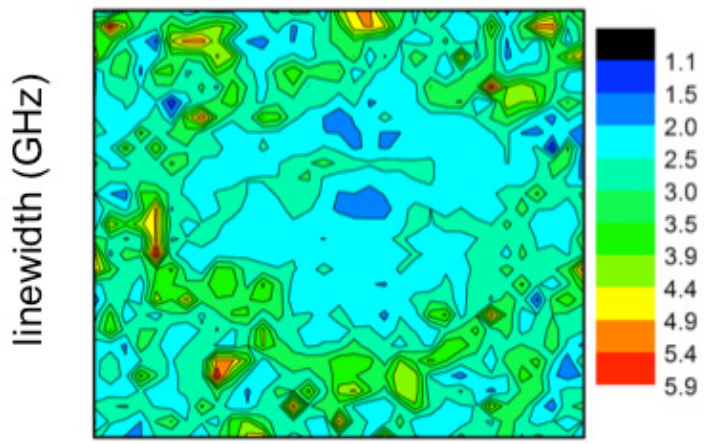

Fig. 8. (Color online) (a) Photomicrograph of a TASTPM mouse brain hippocampal section containing a single plaque collected with the Brillouin microscope in reflection mode using a $20 \times$ objective. (Yellow box denotes a $66 \times 60 \mu \mathrm{m}^{2}$ area where a Brillouin map was acquired using a $1.5 \mu \mathrm{m}$ step-size). Maps based on the (b) frequency shift and (c) linewidth of the Brillouin peak derived from fit analysis of the spectra in the map. Red color region in (b) denotes the plaque core, whilst in (c) it denotes the lipid-loaded ring surrounding the core.

electronic resonances, ${ }^{44}$ so that the relative variation of $\nu^{2}$ gives the correct estimation of the variation in $M^{\prime}$. In our case, assuming $n=1.36^{46}$ and $\rho \sim 1.0$, and taking into account the $1 \%$ frequency shift due to the spread in $q$ (see Sec. 2.2.1), we can calculate the mean variation of the elastic modulus for the probed plaques: it ranges between 9 and $12 \mathrm{GPa}$, for the measured frequency shifts of $\sim 15.4 \mathrm{GHz}$ in the lipid ring and $\sim 17.9 \mathrm{GHz}$ in the core (Figs. 5 and 8 ). 


\section{Conclusion}

We have shown that Brillouin microscopy is a sensitive tool to investigate the micromechanics and viscoelasticity of $\mathrm{A} \beta$ plaques (a major hallmark of AD) within the brain in a mouse model of the disease. Amyloidopathy is characterized by abnormal deposition of $\mathrm{A} \beta$ plaques, which have a rigid core rich in $\mathrm{A} \beta$ protein with a $\beta$-pleated sheet conformation, a viscoelastic lipid-rich layer around the core, and a surrounding heterogeneously composed extracellular matrix, presumably disseminated of glial cell bodies. Correlative micro-Raman analysis of the plaques gives the chemical specificity to identify the molecules responsible for the biomechanical response, hence being able to relate high rigidity to the $\mathrm{A} \beta$ plaque core and low rigidity to the lipid ring. This multimodal approach is key to investigate complex phenomena such as the viscoelasticity of tissues which can provide an invaluable contrast mechanism for the diagnosis of Alzheimer's disease.

\section{Acknowledgments}

This work was generously supported by the Wellcome Trust Institutional Strategic Support Award (WT105618MA). This work was also supported by the Engineering and Physical Sciences Research Council (EP/M028739/1). The TASTPM mice were supplied by Glaxo Smith Kline to the laboratory of Prof Andrew D. Randall. His contribution to this work is acknowledged.

\section{References}

1. M. J. Prince, M. M. Guerchet, M. Prina, The Epidemiology and Impact of Dementia, World Health Organization (2015).

2. J. A. Hardy, G. A. Higgins, "Alzheimer's disease: The amyloid cascade hypothesis," Science $\mathbf{2 5 6}$ (5054), 184-185 (1992).

3. A. D. Randall, J. Witton, C. Booth, A. Hynes-Allen, J. T. Brown, "The functional neurophysiology of the amyloid precursor protein (APP) processing pathway," Neuropharmacology 59(4-5), 243-267 (2010).

4. J. J. Palop, J. Chin, E. D. Roberson, J. Wang, M. T. Thwin, N. Bien-Ly, J. Yoo, K. O. Ho, G. Q. Yu, A. Kreitzer, S. Finkbeiner, J. L. Noebels, L. Mucke, "Aberrant excitatory neuronal activity and compensatory remodeling of inhibitory hippocampal circuits in mouse models of Alzheimer's disease," Neuron 55(5), 697-711 (2007).

5. J. T. Brown, J. Chin, S. C. Leiser, M. N. Pangalos, A. D. Randall, "Altered intrinsic neuronal excitability and reduced $\mathrm{Na}+$ currents in a mouse model of Alzheimer's disease," Neurobiology of aging 32(11), 2109.e1-e14 (2011).

6. T. L. Kerrigan, J. T. Brown, A. D. Randall, "Characterization of altered intrinsic excitability in hippocampal CA1 pyramidal cells of the Abetaoverproducing PDAPP mouse," Neuropharmacology 79, 515-524 (2014).

7. F. Tamagnini, J. Novelia, T. L. Kerrigan, J. T. Brown, K. Tsaneva-Atanasova, A. D. Randall, "Altered intrinsic excitability of hippocampal CA1 pyramidal neurons in aged PDAPP mice," Frontiers in Cellular Neuroscience (2015) 9.

8. F. Tamagnini, S. Scullion, J. T. Brown, A. D. Randall, "Intrinsic excitability changes induced by acute treatment of hippocampal CA1 pyramidal neurons with exogenous amyloid beta peptide," Hippocampus 25(7), 786-797 (2015).

9. F. Palombo, C. P. Winlove, R. S. Edginton, E. Green, N. Stone, S. Caponi, M. Madami, D. Fioretto, "Biomechanics of fibrous proteins of the extracellular matrix studied by Brillouin scattering," J. R. Soc. Interface 11(101), 1-12 (2014).

10. R. S. Edginton, S. Mattana, S. Caponi, D. Fioretto, E. Green, C. P. Winlove, F. Palombo, "Preparation of extracellular matrix protein fibers for Brillouin spectroscopy," J. Vis. Exp. (115), e54648, doi:10.3791/ 54648 (2016).

11. F. Palombo, M. Madami, N. Stone, D. Fioretto, "Mechanical mapping with chemical specificity by confocal Brillouin and Raman microscopy," Analyst 139(3), 729-733 (2014).

12. F. Palombo, M. Madami, D. Fioretto, J. Nallala, H. Barr, A. David, N. Stone, "Chemico-mechanical imaging of Barrett's oesophagus," J. Biophotonics 9(7), 694-700 (2016).

13. G. Scarcelli, S. Besner, R. Pineda, S. H. Yun, "Biomechanical characterization of keratoconus corneas ex vivo with Brillouin microscopy," Invest. Ophthalmol. Vis. Sci. 55(7), 4490-4495 (2014).

14. S. Besner, G. Scarcelli, R. Pineda, S. H. Yun, "In Vivo Brillouin analysis of the aging crystalline lens," Invest. Ophthalmol. Vis. Sci.57(13), 5093-5100 (2016).

15. Z. Steelman, Z. Meng, A. J. Traverso, V. V. Yakovlev, "Brillouin spectroscopy as a new method of screening for increased CSF total protein during bacterial meningitis," J. Biophotonics 8(5), 408-414 (2015).

16. G. Antonacci, R. M. Pedrigi, A. Kondiboyina, V. V. Mehta, R. de Silva, C. Paterson, R. Krams, 
P. Torok, "Quantification of plaque stiffness by Brillouin microscopy in experimental thin cap fibroatheroma," J. R. Soc. Interface 12(112), 1-4 (2015).

17. K. J. Koski, J. L. Yarger, Brillouin imaging, Appl. Phys. Lett. 87(6), 1-3 (2005).

18. G. Scarcelli, S. H. Yun, Confocal Brillouin microscopy for three-dimensional mechanical imaging, Nat. Photon. 2(1), 39-43 (2007).

19. D. Fioretto, L. Comez, G. Socino, L. Verdini, S. Corezzi, P. A. Rolla, "Dynamics of density fluctuations of a glass-forming epoxy resin revealed by Brillouin light scattering," Phys. Rev. E. 59(1), 1899-1907 (1999).

20. L. Comez, C. Masciovecchio, G. Monaco, D. Fioretto, Progress in liquid and glass physics by brillouin scattering spectroscopy, Solid State Physics, E. C. Robert, L. S. Robert, Eds. Vol. 63, pp. 1-77, Academic Press (2012).

21. D. Fioretto, S. Corezzi, S. Caponi, F. Scarponi, G. Monaco, A. Fontana, L. Palmieri, "Cauchy relation in relaxing liquids," J. Chem. Phys. 128(21), 214502 (2008).

22. S. Caponi, S. Corezzi, M. Mattarelli, D. Fioretto, "Stress effects on the elastic properties of amorphous polymeric materials," J. Chem. Phys. 141(21), 214901 (2014).

23. D. R. Howlett, J. C. Richardson, A. Austin, A. A. Parsons, S. T. Bate, D. C. Davies, M. I. Gonzalez, "Cognitive correlates of Abeta deposition in male and female mice bearing amyloid precursor protein and presenilin-1 mutant transgenes," Brain Res. 1017(1-2), 130-136 (2004).

24. D. R. Howlett, K. Bowler, P. E. Soden, D. Riddell, J. B. Davis, J. C. Richardson, S. A. Burbidge, M. I. Gonzalez, E. A. Irving, A. Lawman, G. Miglio, E. L. Dawson, E. R. Howlett, I. Hussain, "Abeta deposition and related pathology in an APP $\mathrm{x}$ PS1 transgenic mouse model of Alzheimer's disease," Histol Histopathol. 23(1), 67-76 (2008).

25. J. T. Mason, T. J. O'Leary, "Effects of formaldehyde fixation on protein secondary structure: A calorimetric and infrared spectroscopic investigation," J. Histochem. Cytochem. 39(1), 225-229 (1991).

26. D. Fioretto, F. Scarponi, "Dynamics of a glassy polymer studied by Brillouin light scattering," Mater. Sci. Eng. A 521-522(0), 243-246 (2009).

27. L. Comez, D. Fioretto, F. Scarponi, G. Monaco, "Density fluctuations in the intermediate glassformer glycerol: A Brillouin light scattering study," J. Chem. Phys. 119(12), 6032-6043 (2003).

28. W. Voigt, "Ueber die Beziehung zwischen den beiden Elasticitätsconstanten isotroper Körper," Annalen der Physik 274(12), 573-587 (1889).

29. A. Reuss, "Berechnung der Fließgrenze von Mischkristallen auf Grund der Plastizitätsbedingung für
Einkristalle," ZAMM - J. Appl. Math. Mech./ Zeitschrift für Angewandte Mathematik und Mechanik 9(1), 49-58 (1929).

30. S. Caponi, A. Fontana, M. Montagna, O. Pilla, F. Rossi, F. Terki, T. Woignier, "Acoustic attenuation in silica porous systems," J. Non-Cryst. Solids 322 (1-3), 29-34 (2003).

31. S. Caponi, P. Benassi, R. Eramo, A. Giugni, M. Nardone, A. Fontana, M. Sampoli, F. Terki, T. Woignier, "Phonon attenuation in vitreous silica and silica porous systems," Philos. Mag. 84(13-16), 1423-1431 (2004).

32. G. Antonacci, M. R. Foreman, C. Paterson, P. Török, "Spectral broadening in Brillouin imaging," Appl. Phys. Lett. 103(22), 221105 (2013).

33. W. Voigt, "Ueber innere Reibung fester Körper, insbesondere der Metalle," Annalen der Physik 283(12), 671-693 (1892).

34. W. Philippoff, Physical Acoustics, W. P. Mason, Ed. Vol. II p. 22, Academic Press, New York (1965).

35. L. Comez, D. Fioretto, L. Palmieri, L. Verdini, P. A. Rolla, J. Gapinski, T. Pakula, A. Patkowski, W. Steffen, E. W. Fischer, "Light-scattering study of a supercooled epoxy resin," Phys. Rev. E. 60(2), 3086-3096 (1999).

36. S. Caponi, G. Carini, G. D'Angelo, A. Fontana, O. Pilla, F. Rossi, F. Terki, G. Tripodo, T. Woignier, "Acoustic and thermal properties of silica aerogels and xerogels," Phys. Rev. B. 70(21), 214204 (2004).

37. C. J. Montrose, V. A. Solovyev, T. A. Litovitz, "Brillouin Scattering and Relaxation in Liquids," $J$. Acoust. Soc. Am. 43(1), 117-130 (1968).

38. A. Battistoni, F. Bencivenga, D. Fioretto, C. Masciovecchio, "Practical way to avoid spurious geometrical contributions in Brillouin light scattering experiments at variable scattering angles," Opt. Lett. 39(20), 5858-5861 (2014).

39. G. Antonacci, S. Braakman, "Biomechanics of subcellular structures by non-invasive Brillouin microscopy," Sci. Rep. 6, 37217 (2016).

40. D. Fioretto, M. Mattarelli, C. Masciovecchio, G. Monaco, G. Ruocco, F. Sette, "Cusp-like temperature behavior of the nonergodicity factor in polybutadiene revealed by a joint light and x-ray Brillouin scattering investigation," Phys. Rev. B. 65(22), 224205 (2002).

41. D. W. Davidson, R. H. Cole, "Dielectric Relaxation in Glycerine," J. Chem. Phys. 18(10), 1417-1417 (1950).

42. L. D. Muiznieks, F. W. Keeley, "Biomechanical design of elastic protein biomaterials: A balance of protein structure and conformational disorder," ACS Biomater. Sci. Eng. (2016).

43. S. Perticaroli, J. D. Nickels, G. Ehlers, A. P. Sokolov, P. Alexei, "Rigidity, secondary structure, and 
the universality of the boson peak in proteins,' Biophys. J. 106(12), 2667-2674 (2014).

44. G. Scarcelli, P. Kim, S. H. Yun, "In Vivo measurement of age-related stiffening in the crystalline lens by brillouin optical microscopy," Biophys. J. 101(6), 1539-1545 (2011).

45. P. Chen, A. Shen, W. Zhao, S. J. Baek, H. Yuan, J. $\mathrm{Hu}$, "Raman signature from brain hippocampus could aid Alzheimer's disease diagnosis," Appl. Opt. 48(24), 4743-4748 (2009).

46. J. Binding, J. Ben Arous, J.-F. Léger, S. Gigan, C. Boccara, L. Bourdieu, "Brain refractive index measured in vivo with high-NA defocus-corrected fullfield OCT and consequences for two-photon microscopy," Opt. Express 19(6), 4833-4847 (2011). 